\title{
Diagnostic accuracy of fine-needle aspiration cytology of the breast in Japan: Report from the Working Group on the Accuracy of Breast Fine-Needle Aspiration Cytology of the Japanese Society of Clinical Cytology
}

\author{
RIN YAMAGUCHI ${ }^{1}$, SHIN-ICHI TSUCHIYA ${ }^{2}$, TAKASHI KOSHIKAWA ${ }^{3}$, AKINORI ISHIHARA $^{4}$, \\ SHINOBU MASUDA ${ }^{5}$, ICHIRO MAEDA ${ }^{6}$, MASAFUMI TAKIMOTO ${ }^{7}$, MASASHI KAWAMOTO $^{8}$, \\ HARUAKI SATOH $^{2}$, MICHIHIKO NARITA ${ }^{9}$, HITOSHI ITOH ${ }^{10}$, TAKASHI KITAMURA ${ }^{11}$, \\ YOSHIKO TSUDA ${ }^{7}$, NAOKI OGANE ${ }^{12}$, EIJI ABE ${ }^{13}$, KATSUHIDE IKEDA $^{14}$, \\ TAKESHI NAKAMURA $^{15}$, HARUMI KAMAGUCHI ${ }^{2}$ and YOSHIRO TOKORO ${ }^{16}$
}

\footnotetext{
${ }^{1}$ Department of Pathology, Kurume University School of Medicine/Kurume University Medical Center, Kurume;

${ }^{2}$ Department of Diagnostic Pathology, Nippon Medical School Hospital, Tokyo; ${ }^{3}$ Department of Pathology,

Aichi Prefectural University, School of Nursing and Health, Aichi; ${ }^{4}$ Department of Clinical Pathology, Matsusaka Chuo General Hospital, Mie; ${ }^{5}$ Department of Pathology, Nihon University School of Medicine, Tokyo;

${ }^{6}$ Department of Pathology, St. Marianna University School of Medicine, Kanagawa; ${ }^{7}$ Department of Pathology, Showa University School of Medicine, Tokyo; ${ }^{8}$ Department of Clinical Pathology, University Hospital, Mizonokuchi, Teikyo University School of Medicine, Kanagawa; ${ }^{9}$ Department of Clinical Pathology, Toyota Kosei Hospital, Aichi;

${ }^{10}$ Division of Diagnostic Pathology, Tokai University Hospital, Kanagawa; ${ }^{11}$ Department of Pathology, Showa University Northern Yokohama Hospital, Kanagawa; ${ }^{12}$ Laboratory for Pathology and Cytology, Kanagawa Cancer Center Hospital, Kanagawa; ${ }^{13}$ Department of Clinical Laboratory,

Kitakyushu Municipal Medical Center, Fukuoka; ${ }^{14}$ Division of Clinical Laboratory, National Cancer Center Hospital, Tokyo; ${ }^{15}$ Department of Pathology, Yokohama Minami Kyousai Hospital, Kanagawa;

${ }^{16}$ Department of Pathology and Molecular Diagnosis, Aichi Cancer Center, Aichi, Japan
}

Received May 18, 2012; Accepted July 10, 2012

DOI: $10.3892 /$ or.2012.2014

\begin{abstract}
The Working Group of the Japanese Society of Clinical Cytology was assembled to assess the current status of breast cytology in Japan by conducting a large-scale survey regarding the accuracy of fine-needle aspiration biopsy (FNAB) in Japan. We collected data and investigated the status of breast cytological diagnosis at 12 different cooperating facilities in Japan, and re-evaluated their falsenegative and false-positive cases. Among 30,535 individuals who underwent a breast cytological examination, analyses were conducted on 10,890 individuals $(35.7 \%)$ in whom cytological diagnoses were confirmed by histology. Among these
\end{abstract}

Correspondence to: Dr Rin Yamaguchi, Department of Pathology, Kurume University School of Medicine, 67 Asahi-machi, Kurume, Fukuoka 830-0011, Japan

E-mail: rin@med.kurume-u.ac.jp

Key words: breast, cytology, diagnostic accuracy, breast cancer screening, accuracy management, fine-needle aspiration biopsy patients, the cytological diagnosis had an inadequate rate of $17.7 \%$, an indeterminate rate of $7.8 \%$, a positive predictive value of 'malignancy suspected' cells of $92.4 \%$, an absolute sensitivity of $76.7 \%$, a complete sensitivity of $96.7 \%$, a specificity of $84.3 \%$, a positive predictive value of 'malignant' cells of $99.5 \%$, a false-negative value of $3.31 \%$, a false-positive value of $0.25 \%$ and an accuracy rate of $88.0 \%$. Subsequently, 297 false-negative and 23 false-positive cases were re-evaluated and several factors were characterized (i.e. histological type, tumor size and misread points). This survey collected data from a large number of cases for breast FNAB. Based on our survey, the accuracy of FNAB in Japan was relatively high compared with the goal of assessment of diagnostic accuracy. However, there were some false-negative and false-positive cases. Improvements in accuracy resulting from the learning points in the present study will lead to more useful and reliable diagnostic tools in clinical practice.

\section{Introduction}

The role of fine-needle aspiration biopsy (FNAB) has been under debate worldwide in recent years (1-5). Needle biopsy has gained popularity and is now considered the standard 
Table I. Collection of data for assessing the diagnostic accuracy of fine-needle aspiration biopsy of the breast.

Histology B3 $(10,890)$

\begin{tabular}{|c|c|c|c|c|c|}
\hline \multirow{3}{*}{$\begin{array}{l}\text { Cytological } \\
\text { category }\end{array}$} & \multirow[b]{3}{*}{ (No.) } & \multirow{2}{*}{\multicolumn{2}{|c|}{ By operation B1 $(8,953)$}} & & \\
\hline & & & & \multicolumn{2}{|c|}{ By needle biopsy B2 $(1,937)$} \\
\hline & & Other than malignancy & Malignancy & Other than malignancy & Malignancy \\
\hline Inadequate & $\begin{array}{c}\mathrm{A} 1 \\
(5,465)\end{array}$ & $\begin{array}{c}\mathrm{C} 1 \\
(153)\end{array}$ & $\begin{array}{c}\text { D1 } \\
(403)\end{array}$ & $\begin{array}{c}\mathrm{E} 1 \\
(361)\end{array}$ & $\begin{array}{l}\mathrm{F} 1 \\
(52)\end{array}$ \\
\hline Normal or benign & $\begin{array}{c}\mathrm{A} 2 \\
(14,538)\end{array}$ & $\begin{array}{l}\mathrm{C} 2 \\
(750)\end{array}$ & $\begin{array}{l}\mathrm{D} 2 \\
(269)\end{array}$ & $\begin{array}{c}\mathrm{E} 2 \\
(796)\end{array}$ & $\begin{array}{l}\mathrm{F} 2 \\
(32)\end{array}$ \\
\hline Indeterminate & $\begin{array}{c}\text { A3 } \\
(2,068)\end{array}$ & $\begin{array}{c}\mathrm{C} 3 \\
(352)\end{array}$ & $\begin{array}{l}\text { D3 } \\
(673)\end{array}$ & $\begin{array}{c}\text { E3 } \\
(241)\end{array}$ & $\begin{array}{l}\text { F3 } \\
(58)\end{array}$ \\
\hline Malignancy suspected & $\begin{array}{c}\text { A4 } \\
(1,146)\end{array}$ & $\begin{array}{c}\mathrm{C} 4 \\
(54)\end{array}$ & $\begin{array}{c}\text { D4 } \\
(695)\end{array}$ & $\begin{array}{c}\mathrm{E} 4 \\
(24)\end{array}$ & $\begin{array}{c}\mathrm{F} 4 \\
(70)\end{array}$ \\
\hline Malignancy & $\begin{array}{c}\mathrm{A} 5 \\
(7,138)\end{array}$ & $\begin{array}{l}\mathrm{C} 5 \\
(35)\end{array}$ & $\begin{array}{c}\text { D5 } \\
(5,569)\end{array}$ & $\begin{array}{l}\text { E5 } \\
(9)\end{array}$ & $\begin{array}{c}\text { F5 } \\
(294)\end{array}$ \\
\hline Total & $\begin{array}{c}\mathrm{A} 6 \\
(30,535)\end{array}$ & $\begin{array}{c}\mathrm{C} 6 \\
(1,344)\end{array}$ & $\begin{array}{c}\mathrm{D} 6 \\
(7,609)\end{array}$ & $\begin{array}{c}\text { E6 } \\
(1,431)\end{array}$ & $\begin{array}{c}\text { F6 } \\
(506)\end{array}$ \\
\hline
\end{tabular}

biopsy method $(1,2)$. On the other hand, it has been discussed that the increase in the number of needle biopsies is not entirely the result of evidence-based reasons (3). There are some advantages of FNAB over needle biopsy for breast lesions: i) it is widely available, easy, quick and inexpensive; ii) it is associated with a lower risk of complications; and iii) it may be appropriate for small lesions, symptomatic (palpable) lesions and for confirming benign lesions (1,3,6-8). Although there is an increasing need for preoperative evaluation of hormone receptors and human epidermal growth factor receptor 2 (HER2/neu) status (1), a number of physicians select needle biopsy in order to avoid obtaining inadequate and/or indeterminate results from cytological analyses (3-5). As a result, the cytological examination has been omitted.

Although FNAB is highly accurate, it is not $100 \%$ accurate (9-15). This point has not been well understood, especially by the general public. However, breast cancer screening is becoming more widespread, and the number of people receiving breast examinations has been increasing. Under these recent circumstances, it is considered that FNAB is an appropriate diagnostic tool $(6,7)$.

The Working Group on the Accuracy of Breast FineNeedle Aspiration Cytology of the Japanese Society of Clinical Cytology was assembled to assess the current status of breast cytology in Japan by conducting a large-scale survey on the accuracy of breast FNAB in 12 cooperating facilities in Japan. Data on 1,250 of the 10,890 subjects in the present study were presented in our previous study as a pilot project (15). However, they were included in this current study to provide a larger sample size, and the data were re-analyzed as part of the larger sample. In the present study, we analyzed and compared the data for cytological diagnosis and individual variables at these 12 facilities, and further investigated the discrepant cases between the cytological and histological diagnoses (false-negative and false-positive cases). It is considered that these data are important for doctors, patients and those in medicolegal circles.

\section{Patients and methods}

We conducted a survey in 12 facilities in order to determine the accuracy of breast FNAB. After reviewing the data from 2009 and the preceding years, we conducted a survey over several years (1-7 years, average 4.3 years) at 12 facilities that dealt with a large number patients. Data were collected from each institution or region, and cytological data confirmed by histological findings obtained after surgery or needle biopsy were included in the study. The manner of data collection is shown in Table I (modified from our previous report with permission) (15).

Classification of cytological samples. In accordance with the General Rules for Clinical and Pathological Recording of Breast Cancer prepared by the Japanese Breast Cancer Society in 2005 (16), individual cytological samples were initially rated as 'inadequate' or 'adequate'. Samples rated as 'adequate' were graded on a four-category scale (16): 'normal/benign'; 'indeterminate' (difficult to distinguish between 'benign' and 'malignant'); 'malignancy suspected'; and 'malignant'. Generally, the cytological diagnostic procedure in Japan involves cytotechnologists initially screening the samples (usually marking the findings on the slides), and then the consultant pathologists making a diagnosis based on the results. The samples are generally extracted from the patients by surgeons, although sometimes by radiologists and/ or general practitioners.

Definitions of variables analyzed and calculations of their diagnostic accuracy. Several terms used in this study merit precise definition and are listed in Table II (modified from 
Table II. Definitions of quality-assurance parameters for cytological examination and calculations of diagnostic accuracy [modified from Table II (15) with permission].

Term

Inadequate value

Indeterminate value

Positive predictive value of 'malignancy suspected' results

Absolute sensitivity

Complete sensitivity

Specificity

Positive predictive value of 'malignant' cells

Negative predictive value of 'normal or benign' cells

False-negative value

False-positive value

Accuracy
Definition and calculation

Percentage of cases whose samples were rated as 'inadequate' among all cases who underwent cell sampling

(A1/A6) x100

Percentage of cases rated as 'indeterminate' among all cases of 'adequate' samples (cases of 'inadequate' samples subtracted from all cases having received cell sampling) $\{$ A3/(A6-A1) $\} \times 100$

Percentage of cases other than false-positive cases among all cases cytologically rated as 'malignancy suspected'

[\{A4-(C4+E4)\}/A4] x100

Percentage of cases cytologically rated as 'malignant' (and confirmed as malignant by histology) among all cases of 'adequate' samples histologically rated as 'malignant' $[(\mathrm{D} 5+\mathrm{F} 5) /\{(\mathrm{D} 6+\mathrm{F} 6)-(\mathrm{D} 1+\mathrm{F} 1)\}] \times 100$

Percentage of cases cytologically rated as 'indeterminate', 'malignancy suspected' or 'malignant' among all cases of 'adequate' samples histologically rated as 'malignant' $[\{(\mathrm{D} 3+\mathrm{F} 3)+(\mathrm{D} 4+\mathrm{F} 4)+(\mathrm{D} 5+\mathrm{F} 5)\} /\{(\mathrm{D} 6+\mathrm{F} 6)-(\mathrm{D} 1+\mathrm{F} 1)\}] \times 100$

Percentage of cases cytologically rated as 'normal or benign' among all cases of 'adequate' samples histologically rated as 'non-malignant'

$[\{\mathrm{A} 2-(\mathrm{D} 2+\mathrm{F} 2)\} /[(\mathrm{A} 6-\mathrm{A} 1)-\{(\mathrm{D} 6+\mathrm{F} 6)-(\mathrm{D} 1+\mathrm{F} 1)\}]] \times 100$

Percentage of cases other than false-positive cases among all cases cytologically rated as 'malignant'

[\{A5-(C5+E5)\}/A5] x100

Percentage of cases other than false-negative cases among all cases cytologically rated as 'normal or benign'

$\{\mathrm{A} 2-(\mathrm{D} 2+\mathrm{F} 2)\} / \mathrm{A} 2\} \times 100$

Percentage of cytologically negative cases among all cases of 'adequate' samples histologically rated as 'malignant'

$[(\mathrm{D} 2+\mathrm{F} 2) /\{(\mathrm{D} 6+\mathrm{F} 6)-(\mathrm{D} 1+\mathrm{F} 1)\}] \times 100$

Percentage of cytologically positive cases among all cases of 'adequate' samples histologically rated as 'non-malignant'

$[(\mathrm{C} 5+\mathrm{E} 5) /[(\mathrm{A} 6-\mathrm{A} 1)-\{(\mathrm{D} 6+\mathrm{F} 6)-(\mathrm{D} 1+\mathrm{F} 1)\}]] \times 100$

Percentage of cases cytologically rated as 'normal or benign' and confirmed as benign by histology and cases cytologically rated as 'indeterminate', 'malignancy suspected' or 'malignant' and confirmed as malignant by histology among all cases of 'adequate' samples histologically rated as 'non-malignant' and 'malignant'

$[\{\mathrm{A} 2-(\mathrm{D} 2+\mathrm{F} 2)+(\mathrm{D} 3+\mathrm{F} 3)+(\mathrm{D} 4+\mathrm{F} 4)+(\mathrm{D} 5+\mathrm{F} 5)\} /(\mathrm{A} 6-\mathrm{A} 1)] \times 100$
Table II in our previous report with permission) (15). These terms are: inadequate value; indeterminate value; positive predictive value of 'malignancy suspected' results; absolute sensitivity; complete sensitivity; specificity; positive predictive value of 'malignant' cells; negative predictive value of 'normal or benign' cells; false-negative value; false-positive value, and accuracy rate. The methods for calculating the diagnostic accuracy of these values based on the recorded columns in Table I are also shown in Table II (15). The combined data for this survey from each institution/region were simply added together. One of the 12 'institutions' in the present study was a region; data were collected and combined from 7 local institutions within the same geographical region, as we previously reported (15). For simplicity and based on the arrangement of the working group conducting this study, these data were considered as representative of 1 'institution' together with data from 11 other institutions. Thus, data obtained from 1,250 histologically confirmed cases from among 5,693 cytologically diagnosed cases from the institutions/regions previously described are included in the present study. 
Analyses of cases showing discrepancies between the cytological and histological diagnoses. Any cases showing discrepancies (i.e., false-negative and false-positive) between the cytological and histological diagnoses were re-analyzed based on histological type, clinical information and tumor size. In addition, these cases were re-evaluated and re-categorized in order to determine the possible reasons for the discrepancies.

\section{Results}

Data from the survey. The data obtained from the individual facilities/regions were summed and calculated. In total, data from 30,535 cases were collected. The cytological diagnosis was established by histological means in 10,890 (35.7\%) of these cases, and this formed the basis for determining the diagnostic accuracy (Table I).

The data were as follows: inadequate rate, $17.7 \%$; indeterminate rate, $7.8 \%$; positive predictive value of 'malignancy suspected' cells, 92.4\%; absolute sensitivity, 76.7\%; complete sensitivity, $96.7 \%$; specificity, $84.3 \%$; negative predictive value of 'normal/benign' cells, $98.2 \%$; positive predictive value of 'malignant' cells, 99.5\%; false-negative value, $3.31 \%$; and false-positive value, $0.25 \%$. The accuracy rate of breast FNAB was $88.0 \%$.

Analyses of cases showing discrepancies between the cytological and histological diagnoses

False-negative cases. There were 301 false-negative cases. Four cases could not be evaluated because we were unable to collect the slides, and therefore a total of 297 cases from the institutions were re-evaluated (including 52 cases from the previous study (17). Histologically, these 297 cases consisted of 94 cases $(31.6 \%)$ of invasive ductal carcinoma (IDC), scirrhous-growing type (SIDC) (16) (Figs. 1 and 2), 70 cases (23.7\%) of ductal carcinoma in situ (DCIS), 40 cases $(13.5 \%)$ of IDC, papillotubular type (16), 22 cases (7.4\%) of IDC, solidtubular type (16), 14 cases $(4.7 \%)$ of invasive lobular carcinoma and several other cases (57 cases including special types, i.e., mucinous carcinoma, apocrine carcinoma).

Regarding the clinical information, there were 59 cases of breast cancer (palpable) (19.9\%), 47 of breast tumor (palpable, but uncertain if benign or malignant) (15.8\%), 38 lacking information (12.8\%) and 30 with abnormal image findings (mammography and/or ultrasound (US) BI-RADS $>3$ b including non-palpable lesions) (10.1\%). In addition, the falsenegative rate for cases with a tumor size or hypoechoic area of US $\leq 1 \mathrm{~cm}$ was $15.5 \%$ (46/297).

After re-evaluating the false-negative cases, the classifications were 20 'inadequate' (6.7\%), 212 'normal/benign' $(71.4 \%)$, 49 'indeterminate' (16.5\%), 11 'malignancy suspected' $(3.7 \%)$ and 5 'malignant' cases $(1.7 \%)$.

The reasons for re-categorization into a new category upon re-evaluation were as follows: 'inadequate' (the small number and/or poor quality of cells made re-evaluation difficult in this category), there were only small clusters in 14 of 20 cases (70\%), and the other 6 cases $(30 \%)$ were composed of small clusters with drying or degeneration; 'normal/benign', 175 of 212 cases $(82.5 \%)$ were benign and/or normal epithelial cells (not atypical, benign small clusters with myoepithelial cells), and the other 37 cases (17.5\%) showed other benign findings (i.e., apocrine metaplasia, foamy cells, fat cells, fibroadenomalike findings); 'indeterminate', 19 of 50 cases $(38.8 \%)$ had clusters of unclear myoepithelial cells, 9 cases $(18.7 \%)$ with a small number of atypical cells presented in the specimens, 5 cases $(10.2 \%)$ showed abundant papillary clusters, 3 cases (6.1\%) showed mild atypia and 14 cases $(26.6 \%)$ were for other reasons; 'malignancy suspected', in 2 of the 11 cases $(18.2 \%)$, atypical cells were present but unclear, atypical cells were present in clusters of unclear myoepithelial cells, and a small number of small atypical cells were present, and 7 cases $(45.5 \%)$ were for other reasons (i.e., the presence of abundant cells, atypical apocrine cells); 'malignancy', myoepithelial cells were absent in clusters in 3 of 5 cases $(60 \%), 1$ case $(20 \%)$ showed cribriform structures and 1 case $(20 \%)$ had isolated atypical cells.

False-positive cases. There were 26 false-positive cases. Three cases could not be evaluated because we were unable to collect the slides; therefore, a total of 22 cases were re-evaluated among all the institutions (including 3 cases from the previous study) (17). Histologically, there were 3 cases each (13.4\%) of papilloma, fibroadenoma, fibrocystic disease (mastopathy) and adenomyoepithelioma (Figs. 3 and 4) and 10 (43.5\%) other cases (i.e., ductal adenoma, epidermal cyst).

Regarding the clinical information, the cases included 6 with abnormal image findings (mammography and/or US BI-RADS >3b with non-palpable lesions) (26.1\%), 5 with breast cancer (palpable) (21.7\%), 5 with breast tumor (palpable, but uncertainty whether benign or malignant) $(21.7 \%), 2$ with breast cancer, suspected $(8.7 \%)$ and 5 others $(21.7 \%)$. In addition, the false-positive rate for cases with a tumor size or hypoechoic area of US $>2 \mathrm{~cm}$ was $39.1 \%(9 / 23)$.

After re-evaluating the false-positive cases, the classifications were 9 'normal/benign' cases (39.1\%), 10 'indeterminate' cases $(43.5 \%)$ and 4 'malignancy suspected' cases (17.4\%). There were no re-classifications as 'inadequate' and 'malignant' cases.

The findings (reasons) for re-categorization into a new category upon re-evaluation were as follows: 'normal/benign', 4 of 9 cases (44.4\%) showed that there were clusters with some myoepithelial cells, 2 cases (22.2\%) showed degenerative chromatic nuclear cells, 2 cases were re-categorized as 'normal/ benign' because the background of degenerative findings was similar to necrotic findings and in 1 case the investigator had insufficient experience in breast disease (normal ductal cells were present); 'indeterminate', 4 of 10 cases (40\%) showed clusters of unclear myoepithelial cells and loose connections between cells, 2 cases each (20\%) showed loosely connected papillary lesions and cribriform-like structures, 1 case showed atypical apocrine cells, and 1 case showed hyperplastic cells; 'malignancy suspected' in each of the 4 cases (25\%), necrosislike findings, atypical apocrine cells, a small number of intracytoplasmic lumina, and low-grade DCIS-like cells were observed. In total, there were no high-grade cells in the falsepositive cases.

\section{Discussion}

To the best of our knowledge, this survey collected data from the largest number of cases for breast FNAB. The role of 

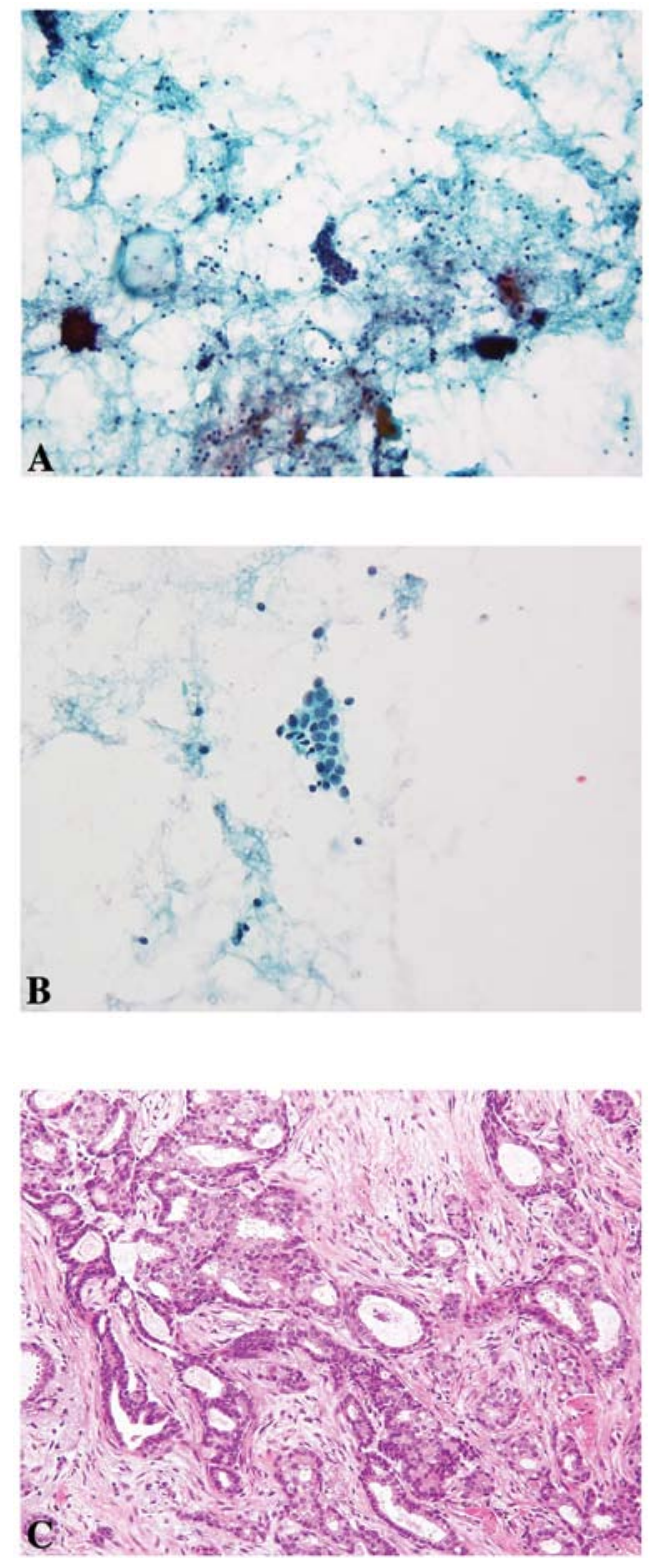

Figure 1. Example of a false-negative case (case record \#24). (A) Small cell clusters were observed with tight connections that appeared benign. The cells were small and relatively monotonous (magnification, x200). (B) In the high-power view, the cells showed enlarged nuclei and myoepithelial cells were unclear (magnification, x400). (C) On an H\&E-stained section from a needle biopsy, the cancer cells showed invasion with irregularly shaped glandular structures with abundant fibrous components. The degrees of pleomorphism and atypia were mild. A diagnosis of invasive ductal carcinoma, papillotubular-scirrhous growing type was made (magnification, x200).

FNAB has been debated recently, as to whether FNAB has been replaced with the core needle (or vacuum) biopsy (2). Consequently, many reports concerning the accuracy of breast cytology have already been discussed, mainly in the 1990s $(9-12,18)$. Although there are a number of advantages and disadvantages for both needle biopsy and FNAB (1), usage of FNAB has been decreasing in many countries over the last decade. Based on our preliminary survey, a number of hospitals and clinics in Japan primarily use FNAB (unpublished data). Therefore, the current accuracy rate of FNAB must be determined and doctors, paramedics, patients as well as medical lawyers must be informed. A cytological examination
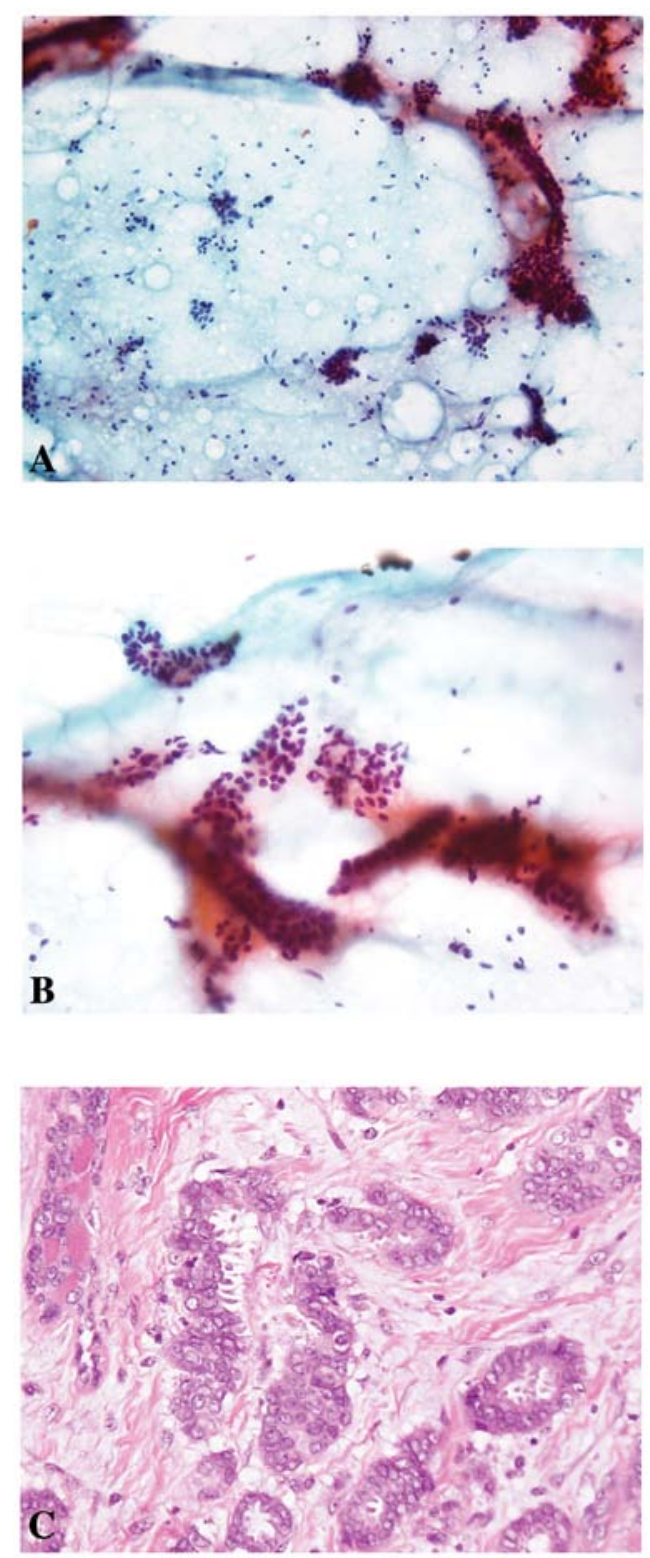

Figure 2. Example of a false-negative case (case record \#29). (A) Several cell clusters with sheet-like structures were noted in naked bipolar myoepithelial nuclei that appeared benign (magnification, x100). (B) However, in the high-power view, the cells had irregular nuclei, and myoepithelial cells in the clusters were unclear (magnification, x400). (C) On an H\&E-stained section from a needle biopsy, a diagnosis of invasive ductal carcinoma, papillotubular-scirrhous growing type was made. The degrees of pleomorphism and atypia were mild to moderate (magnification, $\mathrm{x} 200$ ).

for cancer screening in Japan and in several other countries continues to play an important role. For example, cytological examination plays a major role in the diagnosis of both palpable and non-palpable breast masses in Egypt because it is cost-effective (7).

We compared the combined data from the present study with previously reported data (9-14). The data from the present study were compared with the goals of assessment of diagnostic accuracy reported in the UK $(9,10)$ (absolute sensitivity, $>60 \%$; complete sensitivity, $>80 \%$; specificity, $>60 \%$; positive predictive value, $>95 \%$; false-negative rate, $<5 \%$; false-positive rate, $<1 \%$; inadequate rate, $<25 \%$; suspicious rate, $<20 \%$ ), and were also compared with studies from several other countries, 

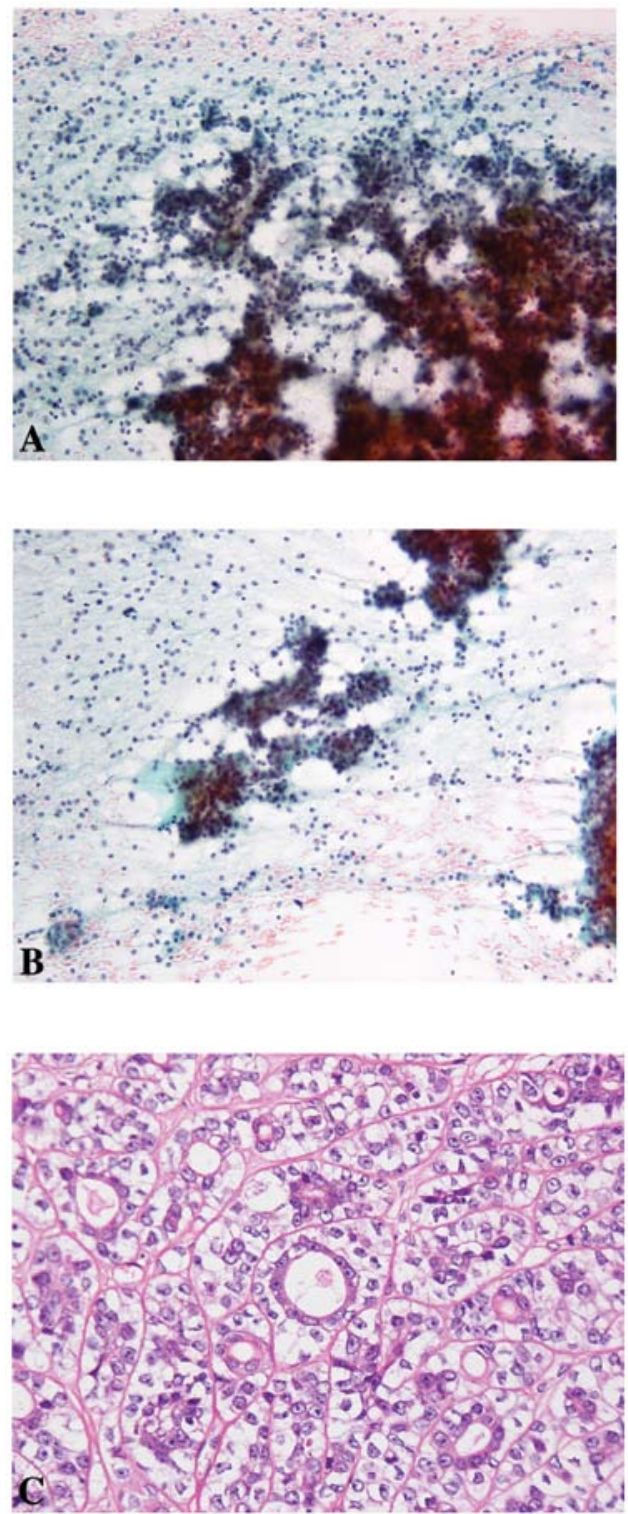

Figure 3. Example of a false-positive case (case record \#21). (A) Abundant naked myoepithelial cell nuclei and various sizes of epithelial cell clusters were noted that appeared malignant (magnification, x200). (B) A loosely cohesive fragment of glandular cell clusters showed highly chromatic nuclei, with light green myxoid stroma (magnification, x400). (C) On an H\&E-stained section, the case was diagnosed as benign adenomyoepithelioma since small glands composed of epithelial cells with eosinophilic cytoplasm were surrounded by myoepithelial cells with a clear cytoplasm (magnification, $\mathrm{x} 400$ ).

including a large-scale study (10,571 cases) in North America (the values had ranges of $75.8-98.7 \%$ for sensitivity, $92-100 \%$ for specificity, $0.6-2.5 \%$ for false-positive rate and $2.5-17.9 \%$ for false-negative rate) $(11,14)$. The combined data in our survey were within these ranges, except for the specificity $(84.3 \%)$ and the inadequate rate $(17.7 \%)$ which were $>10 \%$ (the goal of the Japanese criteria) (16). Therefore, the accuracy of a cytological examination in our survey is considered to be generally useful during clinical practice. However, we previously reported several differences among the institutions in our regions (15), discussed some of the reasons for this situation and speculated on the poor communication between the clinical and pathological sides (15). The present study provided improved results in many categories compared to the previous
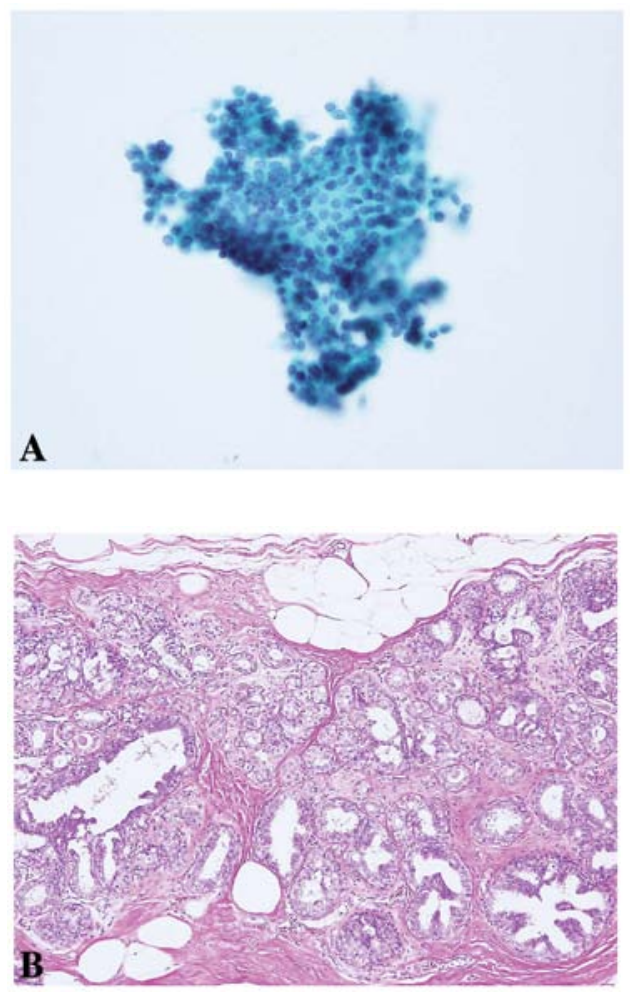

Figure 4. Example of a false-positive case (case record \#14). (A) An epithelial large cluster of ductal cells with mild atypia was noted. The cells were relatively small, but the cells partially overlapped and showed cellular dissociation at the periphery, and myoepithelial cells were absent, which can lead to diagnostic confusion (magnification, x400). (B) On an H\&E-stained section, the case was diagnosed as mastopathy (fibrocystic disease) with usual ductal hyperplasia (magnification, x100).

pilot study. Likely reasons for this are that the previous study included general hospitals and a reference laboratory (15), whereas the present study included mainly larger universitylevel hospitals with specialized breast disease departments.

In addition, we investigated the data with a focus on false-negative and false-positive cases. These values are important because if cases were omitted by the histological diagnosis, false-positive cases would proceed directly to breast cancer surgery, while many false-negative cases may not be re-evaluated. Such results may lead to legal issues. The present data showed that FNAB was not $100 \%$ accurate, and doctors, patients as well as lawyers should be informed. On the other hand, the accuracy of a cytological examination used in order for a diagnosis to be made, was relatively high.

Regarding the 297 false-negative cases, the histology of most cases was SIDC, followed by DCIS. These types of carcinoma are usually detected as small atypical cells in the cytological diagnosis $(17,18)$. After re-evaluation of the 297 false-negative cases, the category of over $90 \%$ cases was changed to 'inadequate', 'normal/benign' or 'indeterminate'. However, the category of 11 other cases was changed to 'malignancy suspected' and that of 5 cases was changed to 'malignancy'. These histological types were mainly SIDC (data not shown; 5/11 cases and 2/5 cases, respectively) and the cells of small clusters showed mild atypia. SIDC showed a tendency toward inadequate and false-negative cytological findings based on their histological characteristics; that is, 
they are usually accompanied by thick fibrous tissues (18). Therefore, when this histological subtype which is simple to detect by imaging (i.e., mammography or ultrasound) is detected, needle biopsy rather than FNAB is recommended (17). Due to the lack of communication between the clinical and pathological sides, clinical information for $15 \%$ of the cases was not available (15). The rate of tumor size under $1 \mathrm{~cm}$ was approximately $15 \%$; thus, the small tumor size was not the main reason for the false-negative cases.

Regarding false-positive cases, the histology of most cases was papilloma, fibroadenoma, fibrocystic disease (mastopathy) or adenomyoepithelioma. Papilloma is one of the most difficult histologic types for cytological diagnosis (19), while fibroadenoma is often misread for hyperplastic epithelial cells (20). Interestingly, adenomyoepithelioma showed the same rate of false-positive cases, for which the histological type has benign to malignant potential and is generally considered a benign lesion (21). This type is comparatively rare, and the frequency of encountering this type is low. However, we should be aware of the type, and of the characteristic cytological findings, including a loosely cohesive fragment of ductal epithelium surrounding fibrous and myxomatous cores (22). The clinical information was based mainly on the abnormal image findings and breast cancer (tumor), and therefore, most cases were suspected of malignancy on the clinical side. The pathological side may have been affected by this information. The rate of tumor size over $2 \mathrm{~cm}$ was more than one-third, and thus the tumor size was often larger. Even after re-evaluation, the category of 4 of 23 cases was still 'malignancy suspected', and these signs were similar to malignant findings. Knowledge of these findings (i.e., apocrine atypia) must be taken into consideration when making a diagnosis.

Although the present study did not always coincide with imaging data, a triple approach (clinical, pathological and radiological) is necessary for accuracy $(1,3)$. Moreover, when the suspected histological type displays difficulties in cytological diagnosis using imaging analysis, which method to select (needle biopsy or cytological examination) depends on individual cases (1). In the future, FNAB should be used for non-palpable lesions with imaging guidance (especially ultrasound) (3).

In conclusion, the accuracy of a cytological examination in Japan is as high as those in other countries, such as the UK and US (9-11). On the other hand, the study demonstrated that there were some false-negative and false-positive cases. Japan may also need to establish a national quality assurance program, similar to the one in the UK $(9,10)$. Furthermore, we must continue to improve accuracy based on the learning points in the present study, so that FNAB can become a more useful and reliable examination in clinical practice.

\section{Acknowledgements}

The authors are indebted to the pathologists and all staff members of the facilities who cooperated in this study. The present study was carried out by the Working Group on the Accuracy of Breast Fine-Needle Aspiration Cytology of the Japanese Society of Clinical Cytology, and the authors would like to thank the members of this highly respected society for their assistance in conducting the study.

\section{References}

1. Tse GM and Tan PH: Diagnosing breast lesions by fine needle aspiration cytology or core biopsy: which is better? Breast Cancer Res Treat 123: 1-8, 2010.

2. Lieske B, Ravichandran D and Wright D: Role of fine-needle aspiration cytology and core biopsy in the preoperative diagnosis of screen-detected breast carcinoma. Br J Cancer 95: 62-66, 2006.

3. Kocjan G, Bourgain C, Fassina A, et al: The role of breast FNAC in diagnosis and clinical management: a survey of current practice. Cytopathology 19: 271-278, 2008.

4. Kocjan G: Fine needle aspiration cytology (Review). Cytopathology 14: 307-308, 2003.

5. Levine T: Breast cytology: is there still a role? Cytopathology 15: 293-296, 2004.

6. Nasuti JF, Gupta PK and Baloch ZW: Diagnostic value and cost-effectiveness of on-site evaluation of fine-needle aspiration specimens: review of 5,688 cases. Diagn Cytopathol 27: 1-4, 2002.

7. Abdel-Hadi M, Abdel-Hamid GF, Abdel-Razek N and Fawzy RK: Should fine-needle aspiration cytology be the first choice diagnostic modality for assessment of all nonpalpable breast lesions? The experience of a breast cancer screening center in Alexandria, Egypt. Diagn Cytopathol 38: 880-889, 2010.

8. Rosa M, Mohammadi A and Masood S: The value of fine needle aspiration biopsy in the diagnosis and prognostic assessment of palpable breast lesions. Diagn Cytopathol 40: 26-34, 2012.

9. Wells CA, Ellis IO, Zakhour HD and Wilson AR: Guidelines for cytology procedures and reporting on fine needle aspirates of the breast. Cytology Subgroup of the National Coordinating Committee for Breast Cancer Screening Pathology. Cytopathology 5: 316-334, 1994.

10. Singh $\mathrm{N}$ and Wells A: Assessment of accuracy in breast cytology. Cytopathology 12: 211-218, 2001.

11. Zarbo RJ, Howanitz PJ and Bachner P: Interinstitutional comparison of performance in breast fine-needle aspiration cytology. A Q-probe quality indicator study. Arch Pathol Lab Med 115: 743-750, 1991.

12. Ciatto S, Bonardi R and Cariaggi MP: Performance of fine-needle aspiration cytology of the breast-multicenter study of 23,063 aspirates in ten Italian laboratories. Tumori 81: 13-17, 1995.

13. Akiu N, Endoh M, Isawa M, et al: Fine needle aspiration cytology of the breast-review of 15470 cases. J Jpn Soc Clin Cytol 46: 323-331, 2007 (In Japanese, abstract in English).

14. Zagorianakou P, Fiaccavento S, Zagorianakou N, Makrydimas G, Stefanou D and Agnantis NJ: FNAC: its role, limitations and perspective in the preoperative diagnosis of breast cancer (Review). Eur J Gynaecol Oncol 26: 143-149, 2005.

15. Yamaguchi R, Tsuchiya S, Koshikawa T, et al: Comparison of the accuracy of breast cytological diagnosis at seven institutions in southern Fukuoka Prefecture, Japan. Jpn J Clin Oncol 42: 21-28, 2012.

16. Sakamoto G, Inaji H, Akiyama F, et al: General rules for clinical and pathological recording of breast cancer. Breast Cancer 12 Suppl: S10-S11, 2005.

17. Yamaguchi R, Tsuchiya S, Koshikawa T, et al: Evaluation of inadequate, indeterminate, false-negative and false-positive cases in cytological examination for breast cancer according to histological type. Diagn Pathol 7: 53, 2012.

18. Park IA and Ham EK: Fine needle aspiration cytology of palpable breast lesions. Histologic subtype in false negative cases. Acta Cytol 41: 1131-1138, 1997.

19. Field A and Mak A: A prospective study of the diagnostic accuracy of cytological criteria in the FNAB diagnosis of breast papillomas. Diagn Cytopathol 35: 465-475, 2007.

20. Yamaguchi R, Tanaka M, Yokoyama T, et al: Cytological features of myxomatous fibroadenoma of the breast. Diagn Cytopathol 40: 316-320, 2012.

21. Tavassoli FA and Soares J: Myoepithelial lesions. In: Pathology and Genetics of Tumours of the Breast and Female Genital Organs. World Health Organization Classfication of Tumours. Tavassoli FA and Devilli P (eds). IARC Press, Lyon, pp86-88, 2003.

22. Ali SZ and Parwani AV: Benign and borderline lesions. In: Breast Cytopathology. Rosenthal DL (ed). Springer, New York, pp57-84, 2007. 